\title{
Integrated Reconfigurable Control and Guidance based on Evaluation of Degraded Performance
}

\author{
H.-S. Shin \\ School of Aerospace, \\ Transport and Manufacturing, \\ Cranfield University, Bedford \\ MK430AL, UK \\ Email: h.shin@cranfield.ac.uk
}

\author{
E. Casaban-Lillo \\ Conekt \\ ZF TRW Technical Centre \\ Stratford Road, Solihull \\ B90 4GW, UK \\ Email: ernest.casaban@zf.com
}

\author{
A. Tsourdos \\ School of Aerospace, \\ Transport and Manufacturing, \\ Cranfield University, Bedford \\ MK430AL, UK \\ Email: a.tsourdos@cranfield.ac.uk
}

\author{
V. Lappas \\ School of Aerospace, \\ Transport and Manufacturing, \\ Cranfield University, Bedford \\ MK430AL, UK \\ Email: vaios.lappas@cranfield.ac.uk
}

\begin{abstract}
The present paper is focused on analysing an integrated reconfigurable control and guidance approach for recovering a small fixed-wing UAV from different actuator faults, which cover locked in place (stuck) and loss of effectiveness. The model of the UAV Aerosonde is used to develop a reconfigurable control system based on the control allocation technique for a variety of faults, such as locked-in-place control surfaces. It is shown through simulation that the developed technique is successful to recover the aircraft from various faults but cannot guarantee success on the planned mission. For mission scenarios where performance degradation is such that the prescribed trajectory cannot be achieved, a reconfigurable guidance system is developed, which is capable of adapting parameters such as the minimum turning radius and the look-ahead distance for obstacle avoidance, to allow the vehicle to dynamically generate a path which guides the aircraft around the no-fly zones taking into account the post-fault reduced performance. Path following is performed by means of a non-linear lateral guidance law and a collision avoidance algorithm is implemented as well. Finally, the integration of control reconfiguration and guidance adaptation is carried out to maximise probabilities of postfailure success in the mission. A methodology is developed, using an error based control allocation parameter, as a measure of performance degradation, which links both reconfiguration and guidance systems. The developed method, although approximate, is proven to be an efficient way of allocating the required degree of reconfiguration in guidance commands when an accurate prediction of the actual performance is not available.
\end{abstract}

\section{INTRODUCTION}

Studies indicate [2] that the UAV industry will continue growing in the next decade, being the highest rate growing sector within the aerospace industry, and reaching spending levels of $\$ 55$ billion. In addition to Military based UAVs, civil UAV applications are growing as a new market possibility for autonomous vehicles. However, a major problem is that in order to use autonomous systems in civilian applications, it is necessary that they fly in civilian airspace, which means that UAVs have to be integrated into the National Airspace System (NAS) of the country where they are flying. This is challenging because every procedure, rule or regulation developed is currently focused on manned aircraft, and for integration to occur, proper standards need to be developed for unmanned systems. Integration of UAVs in NAS depends on them achieving at least an equivalent level of safety to the one that manned aircrafts possess, where the level of safety is defined by the level of risk that UAV activities generate to human life, and other collateral damages that might take place. Figure 1 provides a classification of the accidents that can occur when operating UAVs and their outcomes. It



Fig. 1: Primary/secondary accidents and possible outcomes (from [2])

can be seen, that avoiding mid-air collision, avoiding ground impacts and unintended movements is of great importance, and therefore the approach taken in the work presented in the 
paper about post-failure reconfiguration, aims at maximising the probabilities of successfully recovery when a fault occurs, trying to avoid these kind of incidents, resulting in an increase of reliability in UAS systems and thus contributing at the integration of these in NAS and the growth of civil applications. Furthermore, improving mission performance and probability of completion when an incident has occurred is an important motivation for developing reconfigurable UAV systems.In this paper, we address the integrated control and guidance reconfiguration problem for a small fixed-wing UAV, by designing first a control system that is capable of detecting, when an actuator fault has taken place, if it is possible to recover the aircraft and continue the mission by just reconfiguring control using control allocation or if, the performance degradation induced by the fault is such that it is necessary to change some parameters which will affect the guidance command to ensure that the mission can be continued safely. For this purpose, both systems are designed to be integrated by using output of the reconfigurable control system as an input for the guidance block. This output will serve as a measure of performance degradation and will decide the required degree of reconfiguration for the guidance system.

\section{UAV RECONFIGURABLE CONTROL SYSTEM}

\section{A. UAV Model}

The Aerosonde UAV is a small autonomous airplane designed for weather reconnaissance and remote-sensing missions, collecting data such as temperature, atmospheric pressure, humidity, or wind measurements over oceans and remote areas. The controls differ from those of a conventional aircraft and are shown in Fig. 2. The Aerosonde possesses six control surfaces: left and right flaps $\left(\delta_{f r}\right.$ and $\left.\delta_{f l}\right)$, left and right ailerons $\left(\delta_{a r}\right.$ and $\left.\delta_{a l}\right)$ and left and right ruddervators $\left(\delta_{e r}\right.$ and $\left.\delta_{e l}\right)$. The ruddervators are the inverted $\mathrm{V}$ tail control surfaces and they combine the tasks of elevator and rudder. The relations between the controls of a conventional aircraft and the ones of the Aerosonde UAV are:

$$
\begin{aligned}
\delta_{a} & =\frac{\delta_{a r}-\delta_{a l}}{2} \\
\delta_{f} & =\frac{\delta_{f r}+\delta_{f l}}{2} \\
\delta_{e} & =\frac{\delta_{e r}+\delta_{e l}}{2} \\
\delta_{r} & =\frac{\delta_{e r}-\delta e l}{2}
\end{aligned}
$$

\section{B. Reconfiguration Methods}

Many different approaches to control reconfiguration have been researched and developed. Most of them are based on linear theory and they can be included into one of the next types [6]. A brief description of each big category according to this classification is given in this section. The main categories are: Use of physical redundancy, projecting the fault situation to a previously known scenario, learning control, controller redesign and fault hiding.
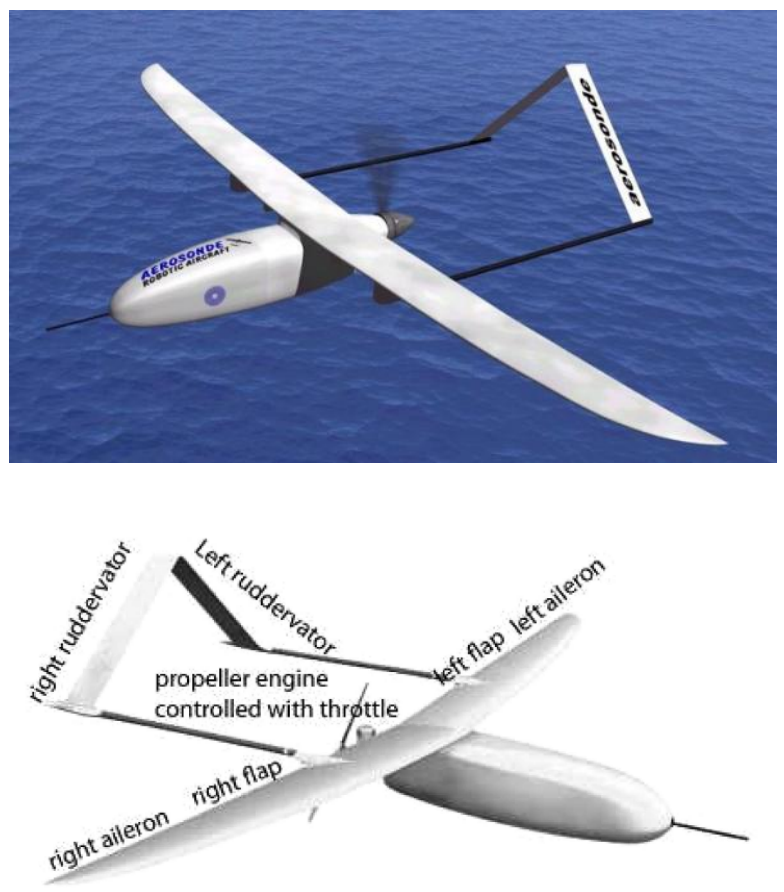

Fig. 2: (a) Image of the UAV Aerosonde (from [4]) (b) Main control surfaces of the UAV Aerosonde (from [5])

Use of physical redundancies refers to actually installing identical hardware components (actuators, sensors). This is a conceptually simple approach to the reconfiguration problem. When a fault is detected, the system switches from the faulty hardware to the healthy redundant component. The problem with this approach is that it is expensive because of the duplication of the components. An advantage is that the computational effort required is very low. It is highly used in applications where safety is a critical matter. Regarding the reconfiguration technique by projection to a known scenario, the methods of this type define the possible faulty situations beforehand and design the controllers. These controllers are organised as banks, each one of them being robust for the specific fault. Usually LQRs are used [7]. When a certain fault takes place, the system tries to find the pre computed scenario that best fits to it and implements on-line the previously off-line designed controller. This approach is conceptually simple but it has the advantages of the offline effort that has to be made to design every controller and the online computational effort to implement each controller. The class of reconfiguration by learning a new control algorithm usually combines a fast component such as a Kalman filter which tries to obtain quick estimates of changing conditions when a fault occurs, and a slower learning component which stores acquired knowledge for future utilisation. This concept is certainly related to adaptive control; however, learning techniques actually use neural networks, expert systems, etc. [8].

For the case of reconfiguration by controller redesign, this means totally redesigning a complete controller after detecting and identifying a fault. For this, the desired behaviour is 
represented by a reference model, and the nominal controller is designed to be close to the closed loop characteristics of this reference model. The last type, reconfiguration by hiding the fault from the nominal controller, implies keeping the nominal controlled without changes after a fault occurs, which might be interesting for some reasons, such as not wanting to change the whole controller when the fault only affects some part of it, or wanting to keep the controller because many iterations have been necessary in its design to keep adapting it. Keeping the nominal controller is possible if a reconfiguration block is put between the former plant and the faulty plant. This reconfiguration block would hide the fault effects from the controller, therefore, from the perspective of the controller, this reconfiguration block forms a reconfigured plant which behaves in the same manner than the nominal one, and there is no need to change it.

\section{INTEGRATED RECONFIGURABLE CONTROL AND GUIDANCE}

This section is focused on the integration of reconfigurable control and guidance and how the integration between both systems works. After establishing the link between both main blocks, a simple approach to take into account uncertainty in sensor measurements will be included, as it is important that the system as a whole proves itself to be robust when facing this issue. A simulation scenario will be set up and different simulations will be run to test and analyse the performance of the full system under different fault conditions. Every simulation is run at a target speed of $V_{c}=25 \mathrm{~m} / \mathrm{s}$ and a constant altitude of $h_{c}=1000 \mathrm{~m}$. As it was demonstrated in Section I, when a fault takes place, the reconfigurable control system redistributes the available control authority to recover the performance to the maximum extent. Every fault implies a certain loss of performance, but if this loss is such that the moments required for the prescribed trajectory cannot be achieved, this will show up by giving a value different from zero in the equation $\xi=B u-v$ from the control allocation algorithm. The higher the performance degradation is after the fault, the higher that the error $\xi$ will be, especially when trying to follow demanding trajectories with sharp turns. Therefore the aim is to relate this error to the performance degradation in order to accordingly reconfigure the guidance system using this parameter. The control allocation algorithm will be used at every moment during the flight, so it is beneficial to take advantage of a parameter that is being constantly calculated, instead of computing performance degradation in an analytical way. Calculating degraded performance in real time would require additional computational effort, as well as preprogrammed methods depending on the fault. Using the error parameter $\xi$, although it is an approximate way of evaluating the performance, it gives an estimated measure and therefore it allows fast and efficient reconfiguration without further calculations. The following equation is going to be assumed for the adaptation of the look-ahead distance depending on $\xi$ :

$$
R_{L A}=R_{L A m i n}+f(\xi)
$$

1) Simulations and Reconfiguration Analysis: Minimum Turning Radius $R_{\min }$ : In this section, a relation between the amount of error from the control allocation block and the achievable minimum turning radius of the aircraft is going to be investigated. By curve fitting expressions are derived, which work for different ranges of faults and that allow to limit the minimum turning radius when this one is reduced by the performance degradation induced by the fault. By knowing the new post-fault limit of the turning capabilities of the aircraft, the guidance block will not try to command some trajectory that is not achievable by the vehicle, as the maximum turn that can be conducted will be limited. In Table I for different stuck positions for the right ruddervator the value of error is shown for a simple trajectory which allows to analyse how the minimum turning radius of the aircraft varies with the change of $\xi$. Fig. 3 shows different trajectories for different

TABLE I: $R_{\min }$ table

\begin{tabular}{ccc}
\hline Stuck position & Error & $R_{\min }(\mathbf{m})$ \\
\hline-20 & -0.31 & 190 \\
-17.5 & -0.24 & 180 \\
-15 & -0.193 & 170 \\
-12.5 & -0.131 & 160 \\
-10 & -0.046 & 140 \\
-7.5 & -0.016 & 100 \\
-5 & 0.06 & 100 \\
-2.5 & 0.1099 & 100 \\
0 & 0.1686 & 130 \\
2.5 & 0.21 & 130 \\
5 & 0.2528 & 150 \\
7.5 & 0.2889 & 165 \\
10 & 0.3228 & 170 \\
12.5 & 0.3566 & 210 \\
15 & 0.39 & 240 \\
17.5 & 0.3925 & NO \\
20 & 0.43 & NO \\
\hline
\end{tabular}

stuck positions of the specified control surface, showing that the maximum curvature of the aircraft, which is given when turning around the corner of the second waypoint, increases with the stuck position for negative values of $\xi$.

\section{A. Simulations for multiple NFZs}

The combined reconfiguration/guidance method is tested in another scenario adding NFZs with different radii to see if the derived relations can be generalised to trajectories and obstacles of different characteristics. The coordinates of the NFZs and the waypoints are shown in Table II. In Figs. 5 and 6 are respectively shown the fault free trajectory in this scenario, and the comparison between the trajectories for the faulty case of right ruddervator stuck. The fault free trajectory, presented for the purpose of comparison with the faulty ones, can be seen to successfully avoid the three NFZs without surpassing the red line. However, looking at Fig. 6, where both trajectories are shown for the case of the right ruddervator stuck at a $-20^{\circ}$ position, it can be seen that given the severity of the fault, the vehicle is struggling to follow the trajectory, and therefore for the case of no reconfiguration applied, it surpasses the red line in two of the NFZs, the first one and 

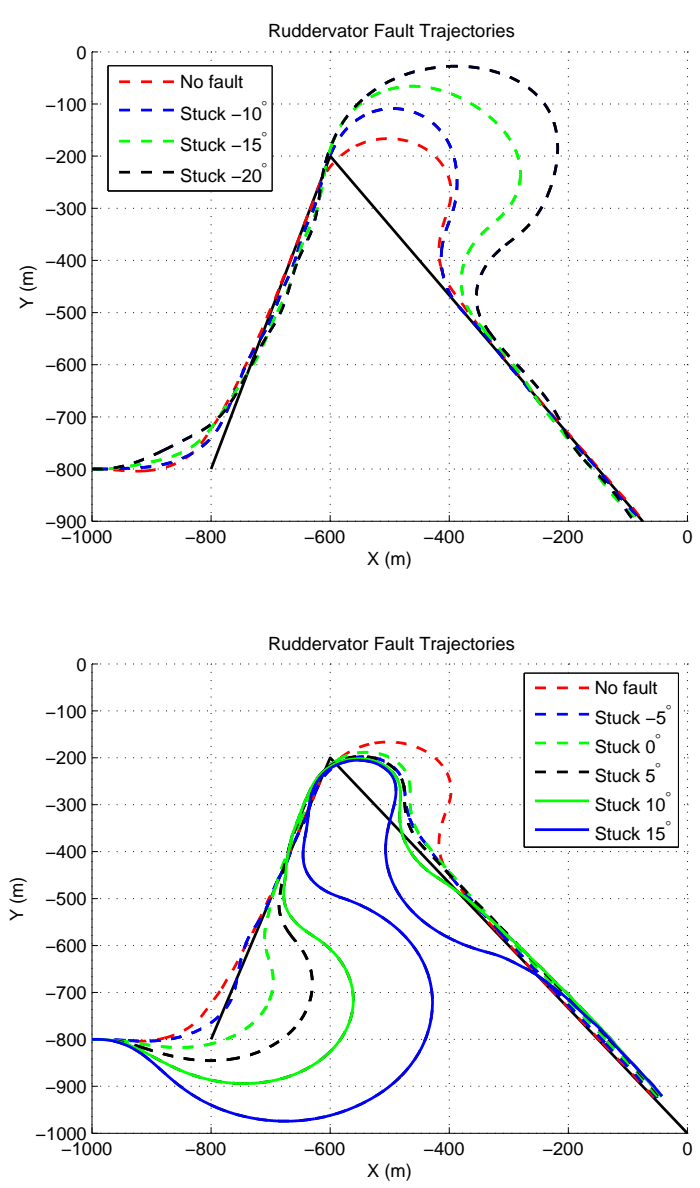

Fig. 3: (a) Minimum turning radius depending on ruddervator fault (negative error) (b) Minimum turning radius depending on ruddervator fault (positive error)

the third one. By reconfiguring the look-ahead distance using the reconfiguration methodology derived, which means adding $0.1 R_{N F Z}$ to the minimum $R_{L A m i n}$, the trajectory shown in blue is obtained, which results in avoiding the three NFZs by starting turns earlier.

TABLE II: Coordinates scenario

\begin{tabular}{cc}
\hline Parameter & Value \\
\hline NFZ1 center & $(0,0) \mathrm{m}$ \\
NFZ1 radius & $300 \mathrm{~m}$ \\
NFZ2 center & $(0,1200) \mathrm{m}$ \\
NFZ2 radius & $150 \mathrm{~m}$ \\
NFZ3 center & $(0,1200) \mathrm{m}$ \\
NFZ3 radius & $100 \mathrm{~m}$ \\
WP1 & $(500,1850) \mathrm{m}$ \\
WP2 & $(900,900) \mathrm{m}$ \\
WP3 & $(-500,1500) \mathrm{m}$ \\
WP4 & $(1000,2000) \mathrm{m}$ \\
\hline
\end{tabular}

It is shown in Fig. 7 the control allocation error for the three axes for NFZ case. It can be seen that it is practically zero (except from some local peaks which are negligible) for the case of the roll axis, as it was expected as a ruddervator fault
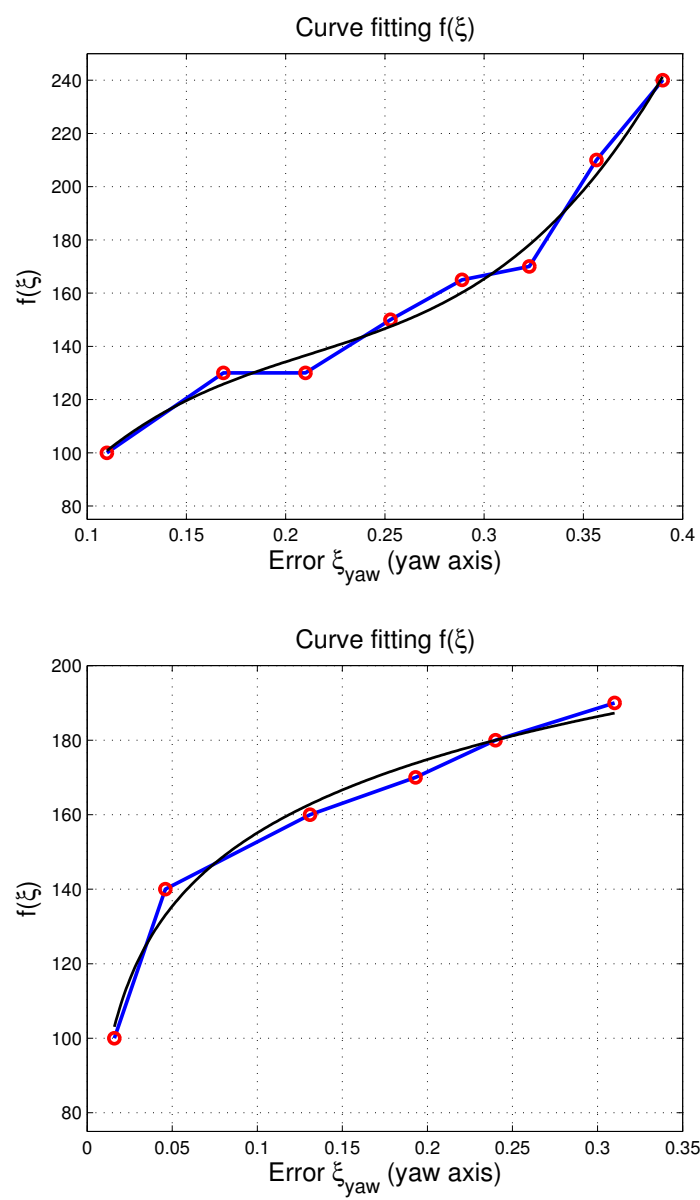

Fig. 4: (a) Minimum turning radius as a function of by curve fitting (positive error) (b) Minimum turning radius as a function of by curve fitting (negative error)

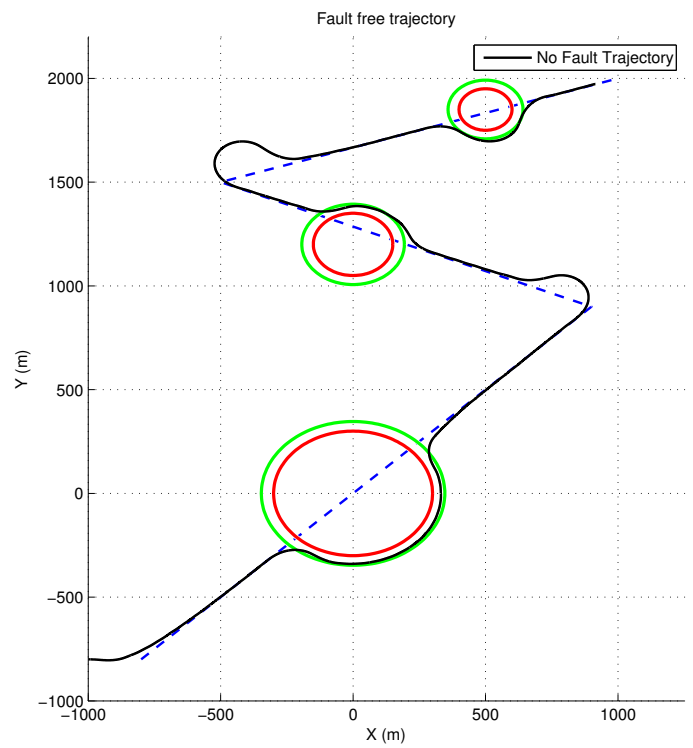

Fig. 5: No fault trajectory for scenario with 3 NFZs (b) Comparison no reconfiguration/ reconfiguration trajectories 


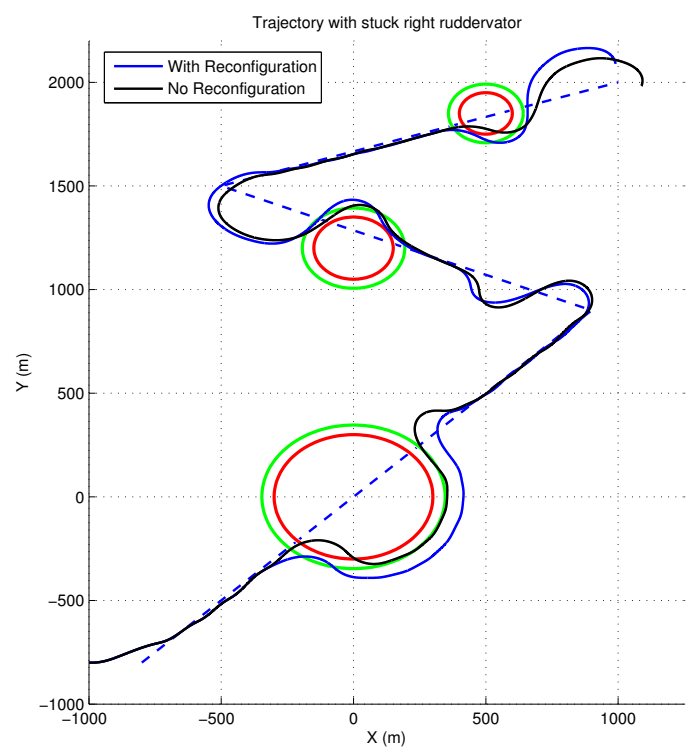

Fig. 6: Comparison no reconfiguration/ reconfiguration trajectories

is being treated. For the pitch and yaw axes the values are the same between them and the mean value is 0.3048 . This supports the assumption made at the beginning of this section for obtaining the expressions of $f(\xi)$ which stated that the mean value for $\xi$ stays approximately the same for a given fault independently of the followed trajectory. As this trajectory is different from the one used to obtain the relations, where a mean value of $\xi_{y a w}=0.283$ was obtained, the assumption, although approximate, can be validated.

\section{CONCLUSION}

The objective of this paper has been to tackle a full integrated reconfigurable control and guidance problem for a small fixed-wing UAV, the Aerosonde UAV, which has some particularities regarding its control surfaces when compared to those of a conventional aircraft. A reconfigurable control system was designed using control allocation techniques based on quadratic programming. The novel fault tolerant control approach proposed aims to, once the fault has been detected and isolated (an ideal FDI system is assumed), distributing control inputs between the remaining healthy control surfaces in order to achieve the required moment that allows the vehicle to follow the prescribed trajectory, or, in the event that this cannot be fully accomplished, minimise the error between the required moment and the achieved one. Novel coupling of both reconfigurable control and guidance systems was implemented in order to detect whether it is possible to recover from an actuator fault by just using the designed control allocation system or if further reconfiguration in the guidance command is needed due to the high performance degradation induced by the fault. The novel reconfiguration approach aims to enable a UAV to successfully continuing its mission. The final results
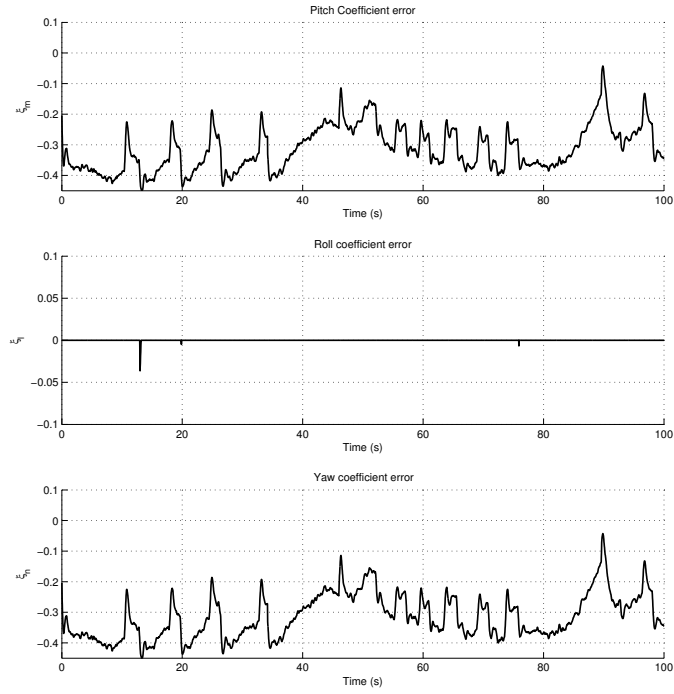

Fig. 7: Allocation error $\xi$ for the case of right ruddervator stuck at $-20^{\circ}$

show that the integrated system is capable of recovering the vehicle from a wide range of actuator faults, taking into account the change in minimum turning radius when sharp turns cannot be achieved by the aircraft because of the fault, and acceptably following the trajectory whenever is possible while flying around obstacles. The guidance system also allows as inputs analytically calculated measures of performance degradation, which will result in a more accurate reconfiguration.

\section{REFERENCES}

[1] World Markets for Military, Civil and Commercial UAVs: Reconnaissance UAVs and Aerial Targets, Frost \& Sullivan, 1998

[2] Dalamagkidis, K, Valavanis, K, \& Piegl, L 2012, On Integrating Unmanned Aircraft Systems Into The National Airspace System [Electronic Resource] : Issues, Challenges, Operational Restrictions, Certification, And Recommendations / By Konstantinos Dalamagkidis, Kimon P. Valavanis, Les A. Piegl, n.p.: Dordrecht : Springer Netherlands, 2012

[3] ASTRAEA. Available on available on http://astraea.aero/. Available on August 13th, 2014.

[4] Aerosonde. Available at http://www.designationsystems.net/dusrm/app4/aerosonde.jpg (August 13th, 2014).

[5] Bateman, F., Noura, H., Ouladsine, M. Fault diagnosis and fault-tolerant control strategy for the aerosonde UAV (2011) IEEE Transactions on Aerospace and Electronic Systems, 47 (3), art. no. 5937287, pp. 21192137.

[6] Lunze, J., Richter, J.H. Reconfigurable fault-tolerant control: A tutorial introduction (2008) European Journal of Control, 14 (5), pp. 359-386.

[7] Aravena J, Zhou K, Li XR, Chowdhury F. Fault tolerant safe flight controller bank. In Proceedings of the 6th IFAC Symposium on Fault Detection, Supervision and Safety of Technical Processes (Safeprocess), pp 859864, Beijing, 2006

[8] Farrell J, Berger T, Appleby B. Using learning techniques to accommodate unanticipated faults. IEEE Control Syst Mag 1993; 13(3): 4049

[9] Stengel RF. Intelligent failure-tolerant control. IEEE Control Syst Mag 1991; 11(4): 1423

[10] Anderson BDO, Moore JB. Optimal control Linear quadratic methods. Prentice Hall, 1989 
[11] Ducard, G., Kulling, K.C., Geering, H.P. Evaluation of reduction in the performance of a small UAV after an aileron failure for an adaptive guidance system (2007) Proceedings of the American Control Conference, art. no. 4282845, pp. 1793-1798.

[12] Park, S., Deyst, J., How, J.P. A new nonlinear guidance logic for trajectory tracking (2004) Collection of Technical Papers - AIAA Guidance, Navigation, and Control Conference, 2, art. no. AIAA 2004-4900, pp. 941-956. 
2016-07-04

\title{
Integrated reconfigurable control and guidance based on evaluation of degraded performance
}

\author{
Shin, Hyo-Sang
}

IEEE

Shin, H.-S. (2016) Integrated reconfigurable control and guidance based on evaluation of degraded performance, 2016 International Conference on Unmanned Aircraft Systems (ICUAS), 7-10 June 2016, Arlington, USA.

http://dx.doi.org/10.1109/ICUAS.2016.7502636

Downloaded from Cranfield Library Services E-Repository 